\title{
Hyperperfusion Immediately after Original Article Reperfusion by Thrombectomy May Predict Hemorrhagic Transformation
}

Tomoyuki Yoshihara, ${ }^{1,2}$ Ryuzaburo Kanazawa, ${ }^{1}$ Hidenori Ohbuchi, ${ }^{3}$ Takanori Uchida, ${ }^{1}$ Tetsuhiro Higashida, ${ }^{1}$ Naoyuki Arai, ${ }^{3}$ Manabu Osakabe, ${ }^{1}$ and Yuichi Takahashi ${ }^{1,3}$

Objective: To evaluate cerebral blood flow immediately after reperfusion following thrombectomy for acute large vessel occlusion, and to examine whether cerebral blood flow after reperfusion is related to hemorrhagic transformation.

Methods: The patients with acute large vessel occlusion in the anterior circulation who obtained reperfusion by thrombectomy were included. Cerebral blood flow was evaluated using single photon emission computed tomography (SPECT) within 6 hours after reperfusion. The patients were divided into two groups with or without hemorrhagic transformation, and basic characteristics, the ratio of cerebral blood flow (affected-side to unaffected-side ratio) in the perfusion area of the middle cerebral artery (MCA asymmetry index), and functional prognosis were compared among two groups.

Results: In all, 23 patients were included; 11 presented hemorrhagic transformation, and 12 did not. The hemorrhagic group exhibited significantly higher MCA asymmetry index than the non-hemorrhagic group (median: 1.54 [1.30-1.83] vs. 1.12 [1.07-1.24], respectively, $P=0.02$ ), and a lower rate tendency of modified Rankin Scale (mRS): 0-2 after 3 months (1 patient [9\%] vs. 6 patients [50\%], respectively, $P=0.06$ ).

Conclusion: In patients with successful reperfusion by thrombectomy, hemorrhagic transformation is predisposed to occur when high MCA asymmetry index is presented. Care should be taken in periprocedural management of blood pressure and sedation for those with high MCA asymmetry index.

Keywords $>$ thrombectomy, acute large vessel occlusion, reperfusion, hyperperfusion, single photon emission computed tomography

\section{Introduction}

Thrombectomy with a stent retriever or aspiration catheter achieved increase in success rate of reperfusion for acute large vessel occlusion, thus contributing to improvement in

${ }^{1}$ Department of Neurosurgery, Nagareyama Central Hospital, Nagareyama, Chiba, Japan

${ }^{2}$ Department of Neurosurgery, Osaka Neurological Institute, Toyonaka, Osaka, Japan

${ }^{3}$ Department of Neurosurgery, Tokyo Women's Medical University Medical Center East, Tokyo, Japan

Received: March 20, 2018; Accepted: October 24, 2018 Corresponding author: Tomoyuki Yoshihara. Department of Neurosurgery, Osaka Neurological Institute, 2-6-23 Shonai, Takaramachi, Toyonaka, Osaka 561-0836, Japan

Email: t-yoshihara@umin.ac.jp

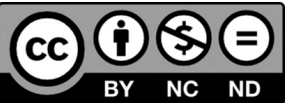

This work is licensed under a Creative Commons Attribution-NonCommercialNoDerivatives International License.

(C)2019 The Japanese Society for Neuroendovascular Therapy functional prognosis. ${ }^{1-3)}$ As to cerebral blood flow after reperfusion, there are several reports with perfusion study or arterial spin labeling (ASL) method using MRI, ${ }^{4,5}$ but few studies have reported cerebral blood flow immediately after endovascular treatment., ${ }^{4,6}$ In this study, cerebral blood flow using single photon emission computed tomography (SPECT) within 6 hours after reperfusion in patients who underwent endovascular treatment for acute large vessel occlusion were evaluated, and the relationship between cerebral blood flow after reperfusion and hemorrhagic transformation was retrospectively examined.

\section{Subjects and Methods}

From January in 2016 to May in 2018, we tried endovascular revascularization for the patients who were diagnosed as acute occlusion of anterior circulation within 12 hours after being last known to be well or symptom onset. The patients were eligible for inclusion in this study if 
reperfusion of grade $2 \mathrm{~B}$ or 3 on thrombolysis in cerebral infarction (TICI) were achieved, and whose resting cerebral blood flow using SPECT were achieved within 6 hours after reperfusion. Those who already had extensive cerebral infarction in responsible perfusion territory on preoperative CT were excluded from the study. This study was approved by the Ethics Review Board of our hospital.

On patients' arrival at the hospital, neurologic deficits that suddenly occurred using the National Institutes of Health Stroke Scale were evaluated, followed by head CT to exclude intracranial hemorrhage. Then, the Alberta Stroke Program Early CT Score-diffusion-weighted imaging (ASPECTS-DWI) by means of 11-point method and occlusion of the internal carotid artery or middle cerebral artery (MCA) was confirmed by DWI and on MRA. If applicable, $0.6 \mathrm{mg} / \mathrm{kg}$ of alteplase was intravenously administered to patients, in reference to "Guidelines for the intravenous application of recombinant tissue-type plasminogen activator (alteplase), the 2nd edition" propounded by the Japan Stroke Society. ${ }^{7)}$ Endovascular revascularization was performed under general anesthesia to control patients' movement and systolic blood pressure below 140 $\mathrm{mmHg}$. In occlusion of MCA, collateral blood flow ${ }^{8)}$ was evaluated by conventional angiography to verify whether the ipsilateral anterior cerebral artery and/or by posterior cerebral artery fed the ischemic area before revascularization. Selection of device for revascularization, such as stent retriever and/or clot aspiration catheter, was left to the operators' decision. After reperfusion, head CT was performed to examine intracranial hemorrhage prior to SPECT study. If revascularization attained to TICI grade of $2 \mathrm{~B}$ or 3 , cerebral blood flow was evaluated by SPECT with ${ }^{123}$ I-N-isopropyl-4-iodoamphethamine within 6 hours after reperfusion. Sedation was used continuously for at least 10 hours after reperfusion with intent to strictly control systolic blood pressure below140 mmHg in the Stroke Care Unit.

Symbia T system (Siemens Japan, Tokyo, Japan) was used for the SPECT scanning. Data were acquired for 30 minutes from 15 minutes after intravenous injection of ${ }^{123}$ I-IMP $222 \mathrm{MBq}$. Regional cerebral blood flow was determined in the automatically defined region of interest per blood-vessel responsible area (anterior cerebral artery area, MCA area: MCA, and posterior cerebral artery area, respectively) on SPECT images with maintained morphological information specific to each subject, which was provided with function of NEUROSTAT ${ }^{9}$ ) run by NEURO FLEXER (Nihon Medi-Physics Co., Ltd., Tokyo, Japan). The ratio of regional cerebral blood flow in the affected side to unaffected side was calculated as asymmetry index. Head CT or MRI was performed on the next day of the procedure or on occasions when neurologic deterioration happened, and we evaluated hemorrhagic transformation in reference to the European-Australasian Acute Stroke Study $\mathrm{II}^{10)}$ and symptomatic hemorrhage in reference to the Safe Implementation of Thrombolysis in Stroke-Monitoring Study (SITS-MOST). ${ }^{11)}$ The functional prognosis was determined by interviewing the patients 3 months after stroke onset, and functional independence was defined as modified Rankin Scale (mRS) score of 0-2.

The patients were divided into the two groups with or without hemorrhagic transformation after reperfusion, and basic characteristics, MCA asymmetry index, and mRS were compared among two groups. The cutoff value of MCA asymmetry index was determined by hemorrhagic transformation. The correlation between ASPECTS-DWI before reperfusion and MCA asymmetry index were also examined.

As a sub-analysis for patients with MCA occlusion, we investigated whether collateral circulation before reperfusion was correlated with MCA asymmetry index. Collateral circulation was evaluated using collateral flow grading proposed by Higashida et al. ${ }^{8)}$

For statistical analysis, BellCurve for Excel software (version 2.15, Social Survey Research Information Co., Ltd., Tokyo, Japan) was used. The numerical value was expressed as median (interquartile range) to the second decimal place. A P value of less than 0.05 was regarded as significant. The variables were compared between the two groups using the Mann-Whitney U-test and Fisher's exact probability test. A cutoff value was defined as a point to figure the shortest distance from the left upper corner on a receiver operating characteristic (ROC) curve. On occasions when MCA asymmetry index exceeds the cutoff value, the odds ratio of hemorrhagic transformation was figured out by $2 \times 2$ table, and verified by Fisher's exact probability test (paired test). Concerning the correlation, Spearman's rank correlation coefficient was calculated, and significance was tested.

\section{Results}

Of the 645 consecutive patients with stroke who were brought to our hospital during the study period, we performed revascularization for 98 patients who arrived less than 12 hours after onset with large vessel occlusion confirmed by MRI. In all, 82 patients with occlusion of anterior 
Table 1 Patient characteristics

\begin{tabular}{|c|c|c|c|c|}
\hline & \multirow{2}{*}{ Total } & \multicolumn{2}{|c|}{ Hemorrhagic transformation } & \multirow{2}{*}{$P$ value } \\
\hline & & $(+)$ & $(-)$ & \\
\hline Variable & $\mathrm{n}=23$ & $\mathrm{n}=11$ & $\mathrm{n}=12$ & - \\
\hline Age, median (year, IQR) & $75(65-79)$ & $72(65-77)$ & $75(67-81)$ & 0.49 \\
\hline Female sex, number (\%) & $11(47)$ & $4(36)$ & $7(58)$ & $0.41^{*}$ \\
\hline Internal carotid artery occlusion, number (\%) & $11(47)$ & $7(66)$ & $4(33)$ & $0.22^{*}$ \\
\hline NIHSS, median (IQR) & $20(12-22)$ & $22(15-22)$ & $18(10-20)$ & 0.27 \\
\hline ASPECTS-DWI, median (IQR) & $5(4-8)$ & $5(2-6)$ & $7(4-9)$ & 0.16 \\
\hline Intravenous injection of alteplase, number (\%) & $4(17)$ & $3(27)$ & $1(8.3)$ & $0.31^{*}$ \\
\hline $\begin{array}{l}\text { Time from last well known to reperfusion, } \\
\text { median (min, IQR) }\end{array}$ & $317(280-543)$ & $382(290-503)$ & $312(278-589)$ & 0.95 \\
\hline $\begin{array}{l}\text { Time from reperfusion to SPECT, } \\
\text { median (min, IQR) }\end{array}$ & $178(130-203)$ & $200(132-212)$ & $167(127-189)$ & 0.19 \\
\hline MCA asymmetry index, median (IQR) & $1.24(1.11-1.55)$ & $1.54(1.30-1.83)$ & $1.12(1.07-1.24)$ & 0.02 \\
\hline $\begin{array}{l}\text { Modified Rankin Scale; } 0-2 \text { at } 3 \text { months, } \\
\text { number (\%) }\end{array}$ & $7(30)$ & $1(9)$ & $6(50)$ & $0.06^{*}$ \\
\hline
\end{tabular}

${ }^{*} \mathrm{P}$ value was calculated with the use of Fisher's exact probability test. The Mann-Whitney U-test was used for the other variables without asterisk. ASPECTS-DWI: Alberta stroke program early CT score - diffusion weighted imaging; IQR: interquartile range; MCA: middle cerebral artery; NIHSS: National Institutes of Health Stroke Scale; SPECT: single photon emission computed tomography

circulation were included (26: internal carotid artery; 56: MCA). While SPECT study was performed in 28 from 59 patients who achieved TICI $2 \mathrm{~B}$ or 3 , we finally analyzed 23 patients, excluding 5 whose SPECT study was not performed within 6 hours after reperfusion (Table 1). In all, 11 patients had internal carotid artery occlusion, whereas the remaining12 were occlusion of MCA. Alteplase was intravenously administered to four patients. The median interval time from last known to be well or symptom onset to reperfusion was 317 minutes (280-543). The median time from reperfusion to SPECT was 178 minutes (130-203). No patient with the achievement of TICI 2B or 3 showed any hemorrhagic transformation on CT prior to SPECT. In all, 11 patients showed hemorrhagic transformation on CT after the next day of reperfusion although they had no symptomatic hemorrhage defined by SITS-MOST. MCA asymmetry index was significantly higher in the hemorrhagic group than in the non-hemorrhagic group (1.54 [1.30-1.83] vs. 1.12 [1.07-1.24], respectively, $\mathrm{P}=0.02$ ). The rate of patients with mRS score of 0 to 2 after 3 months was predisposed to be lower in the hemorrhagic group than in the no-hemorrhagic group ( 1 patient $[9 \%$ ] vs. 6 patients $[50 \%$ ], respectively, $\mathrm{P}=$ 0.06 ) although there was no significant difference. The cutoff value of MCA asymmetry index was 1.24 , derived from the ROC curve (Fig. 1A, area under the curve: 0.78, sensitivity: 0.81/specificity: 0.75 ). The odds ratio for hemorrhagic transformation was 13.5 (95\% confidence interval: 1.8-101.1, P= 0.012 ), if MCA asymmetry index was more than 1.24.

There was a significantly negative correlation between ASPECTS-DWI and MCA asymmetry index (Spearman's rank correlation coefficient: $r_{s}=-0.68, P<0.001$, Fig. 1B): a patient with lower ASPECTS-DWI was followed by higher MCA asymmetry index after achievement of reperfusion. A restricted analysis of 12 patients with occlusion of MCA presented a significantly negative correlation between collateral flow grade and MCA asymmetry index $\left(r_{s}=-0.71, P=0.008\right.$, Fig. 2), which represented that the worse collateral flow tended to lead the higher MCA asymmetry index.

A representative case is shown in Fig. 3.

\section{Discussion}

To our investigation, there were only a few studies to evaluate cerebral blood flow after achievement of reperfusion by thrombectomy for acute large vessel occlusion. Kneihsl et al. ${ }^{6}$ evaluated the mean blood flow of MCA using transcranial Doppler ultrasonography in average 6 hours after reperfusion at TICI grade $2 \mathrm{~B}$ or 3 . Nael et al.4) reported the evaluation of cerebral blood flow using ASL method within 7 hours after reperfusion while they put emphasis on the occasional underestimation of hyperperfusion in comparison with a conventional method with contrast medium. In this study, we assessed cerebral blood flow using SPECT within 6 hours after reperfusion, which was earlier than in the other studies above. We conclude that the higher MCA asymmetry index may lead to hemorrhagic transformation more frequently. There are two noteworthy reports on hyperperfusion after carotid artery stenting. Ogasawara et al. ${ }^{12)}$ reported that hyperperfusion in most cases occurred within 12 hours after carotid artery stenting. Kaku et al. ${ }^{13)}$ evaluated cerebral blood flow using SPECT immediately after carotid artery stenting, and indicated that the asymmetry index ranged from 1.11 to 1.20 in patients with hyperperfusion. 


\section{ROC curve}
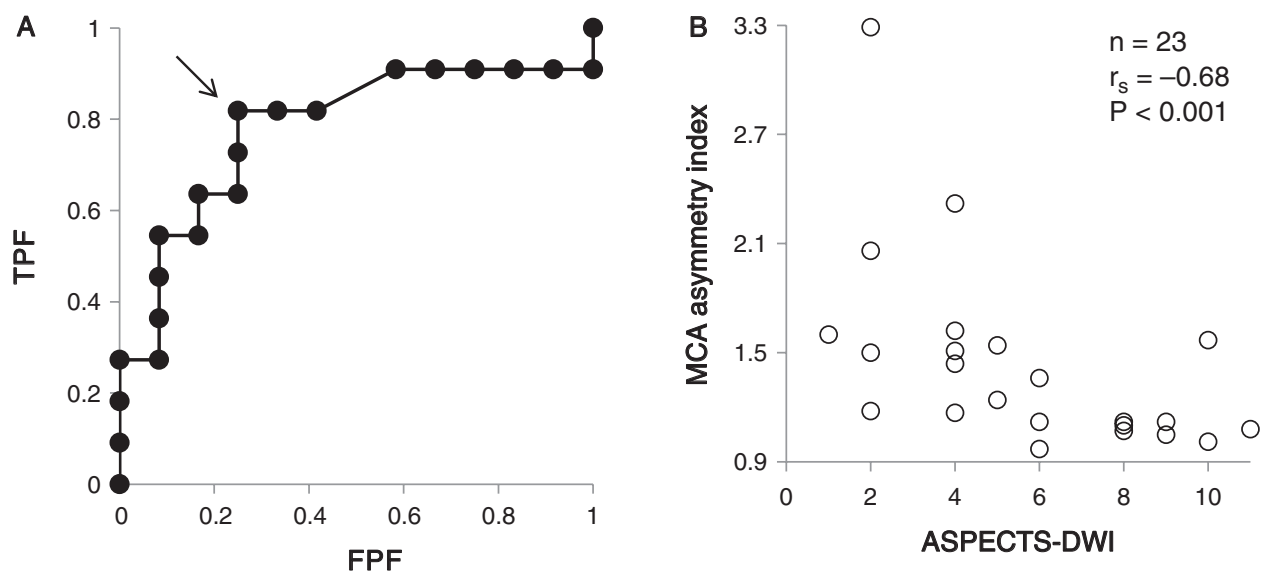

Fig. 1 (A) The ROC curve showed whether hemorrhagic transformation after reperfusion was predictable by the ratio of regional cerebral blood flow in the perfusion area responsible for the MCA (affected side/ unaffected side; MCA asymmetry index). The arrow indicates a cutoff point. (B) Scatter diagram showed the correlation between ASPECTS-DWI and MCA asymmetry index. ASPECTS-DWI: Alberta Stroke Program Early CT Score-diffusion-weighted imaging; FPF: false-positive fraction; MCA: middle cerebral artery; $r_{s}$ : Spearman's rank correlation coefficient; ROC: receiver operating characteristic; TPF: true-positive fraction

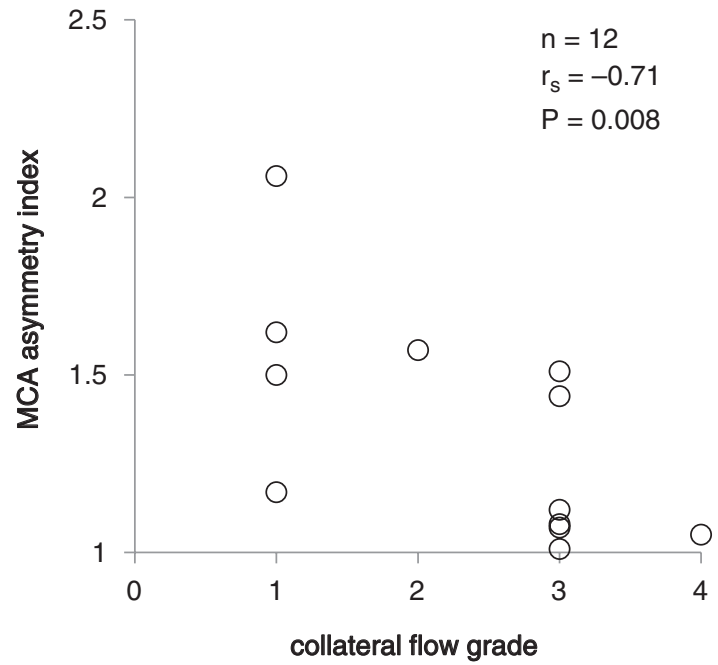

Fig. 2 In an analysis for 12 patients with acute occlusion of MCA, scatter diagram showed the correlation between collateral flow grade and MCA asymmetry index. Collateral flow grade before reperfusion was defined at 5 grades (Grade $0-4){ }^{8)}$ MCA asymmetry index: the ratio of cerebral blood flow in the MCA perfusion area after reperfusion. MCA: middle cerebral artery; $r_{s}$ : Spearman's rank correlation coefficient

Hashimoto et al. ${ }^{14)}$ reported a case of hyperperfusion syndrome presenting symptomatic intracranial hemorrhage 12 hours after reperfusion therapy for acute occlusion of internal carotid artery and MCA. Backhaus et al. ${ }^{15)}$ presented a case of hyperperfusion syndrome the next day after thrombectomy for acute MCA occlusion. As indicated in this study, some patients with acute large vessel occlusion show hyperperfusion within 6 hours after reperfusion although no hemorrhagic transformation is observed at all immediately after reperfusion. Low ASPECTS-DWI might be considered to lead to an increase in MCA asymmetry index after reperfusion. While limited analysis for occlusion of MCA, unfavorable collateral blood flow might also be apt to increase in MCA asymmetry index. In case of presenting high MCA asymmetry index after reperfusion, late hemorrhagic transformation which can cause poor functional prognosis may take place. Therefore, it is considerable to adequately control blood pressure ${ }^{16)}$ and sedation ${ }^{17)}$ during the periprocedural period, especially, if reperfusion is achieved. Adequate periprocedural management as listed above possibly influenced the preferable results in this study, in which nearly one half of the patients presented hemorrhagic transformation without any symptom.

Our study has some limitations. This retrospective analysis for a small number of subjects was possible to identify no independent factor of hemorrhagic transformation. In addition, this study did not at all deny the involvement of ASPECTS-DWI or collateral blood flow as confounding factor of hemorrhagic transformation. In resolving such problems, it is necessary to plan to verify a larger number of subjects in a prospective-controlled clinical study. Another limitation is insufficiency of evaluation for collateral blood flow. Especially, in acute occlusion of the internal carotid artery, it is often laborious to perform angiography in other vessels to assess collateral blood flow, which was a weak point in this study. An alternative modality, such as $\mathrm{CT}$ angiography, ${ }^{18)}$ which can evaluate collateral blood flow regardless 

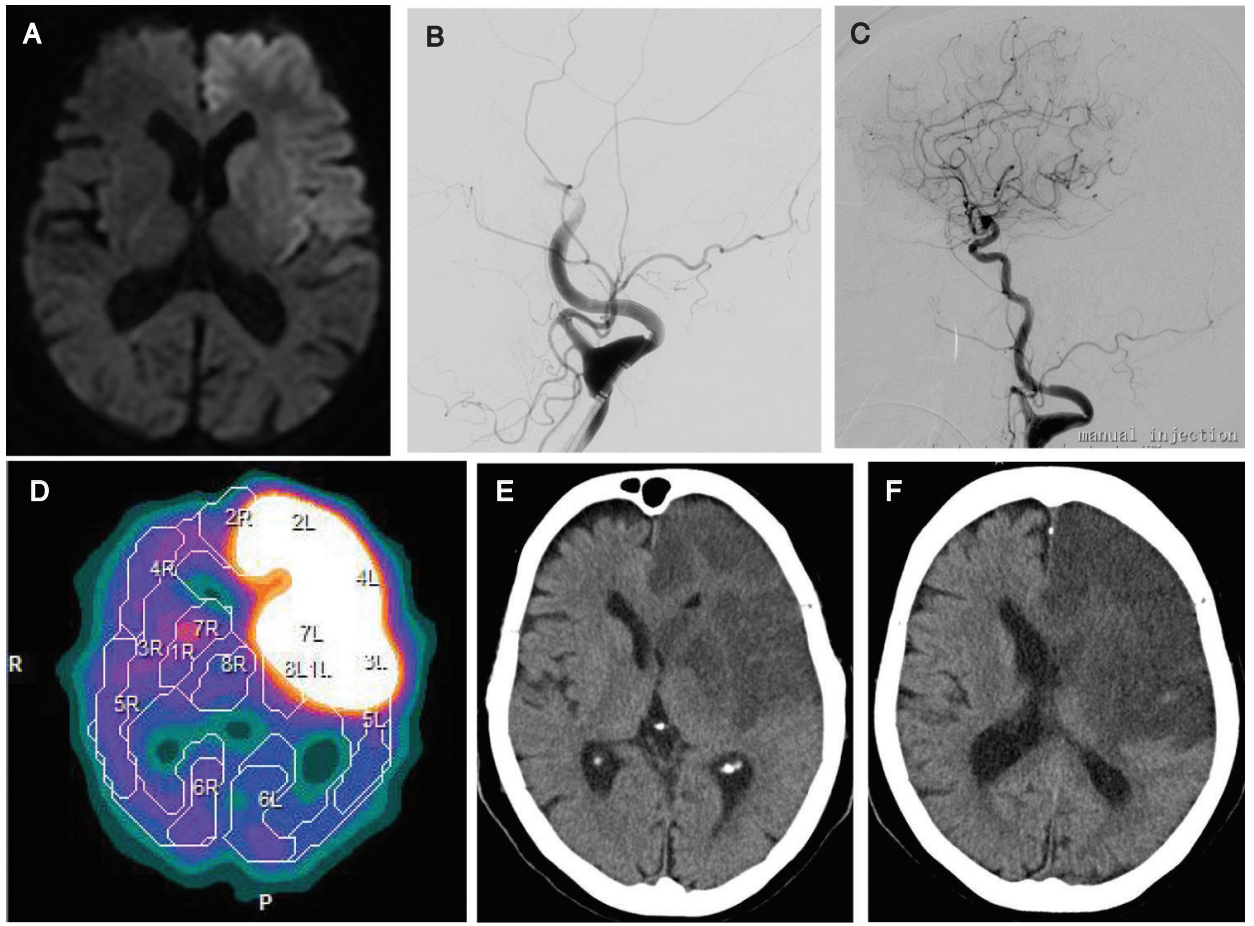

Fig. 3 A 75-year-old female with a history of atrial fibrillation was taking orally $10 \mathrm{mg}$ of rivaroxaban and presented a sudden right hemiplegia and aphasia. On arrival at the hospital, diffusion-weighted MRI showed high intensity in the left cortex of the frontal lobe and basal ganglia (A). Left common carotid angiography (lateral view) demonstrated occlusion of the internal carotid artery (B). Thrombectomy led to complete reperfusion (C). Single photon emission CT after reperfusion revealed hyperperfusion mainly in the left frontal lobe (D). CT on the next day of thrombectomy did not show marked hemorrhage $(E)$, but there was a hemorrhagic transformation on day $3(\mathbf{F})$.

of the occlusion site, might elucidate relation between collateral blood flow and hyperperfusion after reperfusion.

\section{Conclusion}

We had evaluated cerebral blood flow within 6 hours after endovascular revascularization for acute large vessel occlusion in anterior circulation. The MCA asymmetry index was significantly higher in the hemorrhage group than in the non-hemorrhage group, and the functional prognosis after 3 months was predisposed to being unfavorable in the former. Careful control of blood pressure and sedation in periprocedural period should be taken if reperfusion is achieved for those with high MCA asymmetry index to avoid hemorrhagic transformation.

\section{Acknowledgment}

We thank the staff of the Department of Radiology, Nagareyama Central Hospital, and Ms. Sumie Toda for their cooperation.

\section{Disclosure Statement}

There is no conflict of interest for the first author and coauthors.

\section{References}

1) Berkhemer OA, Fransen PS, Beumer D, et al: A randomized trial of intraarterial treatment for acute ischemic stroke. N Engl J Med 2015; 372: 11-20.

2) Campbell BC, Mitchell PJ, Kleinig TJ, et al: Endovascular therapy for ischemic stroke with perfusion-imaging selection. N Engl J Med 2015; 372: 1009-1018.

3) Goyal M, Demchuk AM, Menon BK, et al: Randomized assessment of rapid endovascular treatment of ischemic stroke. N Engl J Med 2015; 372: 1019-1030.

4) Nael K, Meshksar A, Liebeskind DS, et al: Periprocedural arterial spin labeling and dynamic susceptibility contrast perfusion in detection of cerebral blood flow in patients with acute ischemic syndrome. Stroke 2013; 44: 664-670.

5) Okazaki S, Yamagami H, Yoshimoto T, et al: Cerebral hyperperfusion on arterial spin labeling MRI after reperfusion 
therapy is related to hemorrhagic transformation. $J$ Cereb Blood Flow Metab 2017; 37: 3087-3090.

6) Kneihsl M, Niederkorn K, Deutschmann H, et al: Increased middle cerebral artery mean blood flow velocity index after stroke thrombectomy indicates increased risk for intracranial hemorrhage. J Neurointerv Surg 2018; 10: 882-887.

7) Minematsu K, Toyoda K, Hirano T, et al: Guidelines for the intravenous application of recombinant tissue-type plasminogen activator (alteplase), the second edition, October 2012: a guideline from the Japan Stroke Society. J Stroke Cerebrovasc Dis 2013; 22: 571-600.

8) Higashida RT, Furlan AJ, Roberts H, et al: Trial design and reporting standards for intra-arterial cerebral thrombolysis for acute ischemic stroke. Stroke 2003; 34: e109-137.

9) Ogura T, Hida K, Masuzuka T, et al: An automated ROI setting method using NEUROSTAT on cerebral blood flow SPECT images. Ann Nucl Med 2009; 23: 33-41.

10) Larrue V, von Kummer RR, Müller A, et al: Risk factors for severe hemorrhagic transformation in ischemic stroke patients treated with recombinant tissue plasminogen activator: a secondary analysis of the European-Australasian Acute Stroke Study (ECASS II). Stroke 2001; 32: 438-441.

11) Wahlgren N, Ahmed N, Dávalos A, et al: Thrombolysis with alteplase for acute ischaemic stroke in the Safe Implementation of Thrombolysis in Stroke-Monitoring Study (SITS-MOST): an observational study. Lancet 2007; 369: 275-282.

12) Ogasawara K, Sakai N, Kuroiwa T, et al: Intracranial hemorrhage associated with cerebral hyperperfusion syndrome following carotid endarterectomy and carotid artery stenting: retrospective review of 4494 patients. J Neurosurg 2007; 107: 1130-1136.

13) Kaku Y, Yoshimura S, Kokuzawa J: Factors predictive of cerebral hyperperfusion after carotid angioplasty and stent placement. AJNR Am J Neuroradiol 2004; 25: 1403-1408.

14) Hashimoto $T$, Matsumoto $S$, Ando $M$, et al: Cerebral hyperperfusion syndrome after endovascular reperfusion therapy in a patient with acute internal carotid artery and middle cerebral artery occlusions. World Neurosurg 2018; 110: 145-151.

15) Backhaus R, Boy S, Fuchs K, et al: Hyperperfusion syndrome after MCA embolectomy - a rare complication? Am J Case Rep 2013; 14: 513-517.

16) Abou-Chebl A, Reginelli J, Bajzer CT, et al: Intensive treatment of hypertension decreases the risk of hyperperfusion and intracerebral hemorrhage following carotid artery stenting. Catheter Cardiovasc Interv 2007; 69: 690-696.

17) Kaisti KK, Långsjö JW, Aalto $\mathrm{S}$, et al: Effects of sevoflurane, propofol, and adjunct nitrous oxide on regional cerebral blood flow, oxygen consumption, and blood volume in humans. Anesthesiology 2003; 99: 603-613.

18) Lima FO, Furie KL, Silva GS, et al: The pattern of leptomeningeal collaterals on CT angiography is a strong predictor of long-term functional outcome in stroke patients with large vessel intracranial occlusion. Stroke 2010; 41: 2316-2322. 